\title{
Influencia de componentes de inteligencia emocional en rendimiento académico escolar: Análisis en una Institución Educativa en Perú
}

\section{Influence of emotional intelligence components on academic school performance: Analysis in an educational institution in Peru}

\author{
DOI: $10.46932 / \mathrm{sfjdv2n5-053}$
}

Received in: Oct 1st, 2021

Accepted in: Dec 30th, 2021

\section{Juan Guillermo Lazo Lazo}

Es profesor a tiempo completo del Departamento Académico de Ingeniería de la Universidad del Pacífico. Es Doctor (Ph.D.) en Ingeniería Eléctrica en el área de Métodos de Apoyo a la Decisión y

Master of Science en Ingeniería Eléctrica en el área de Sistemas de Computación ambos por la Pontificia Universidad Católica de Río de Janeiro (Brasil). Fue docente de pregrado y postgrado en la Pontificia Universidad Católica de Río de Janeiro, en el Centro Federal de Educación Tecnológica Celso Suckow da Fonseca - CEFET/Rio de Janeiro, y en el programa doctoral en Ciencias de Computación de la Universidad Nacional de San Agustín de Arequipa. Es investigador, coordinador y líder de proyectos de investigación e innovación científica y soluciones de apoyo para la toma de decisiones estratégicas desarrolladas para empresas brasileñas.

E-mail:.jg.lazol@up.edu.pe

\section{Suely Noronha de Oliveira}

Formada en Pedagogía por la Universidad Federal de Viçosa (Minas Gerais - Brasil), Maestría en Educación por la Pontificia Universidad Católica de Rio de Janeiro. Asesora de tesis en la Escuela de Posgrado Maestría en Gestión de Instituciones Educativas de la Universidad Antonio Ruiz de Montoya

(Perú). Miembro del Grupo de Investigación Ciudadanía, Educación y Diversidad de la Facultad de

Educación (PUCP). Profesora de la Facultad de Educación de la UFRJ hasta diciembre/2016. Fue coordinadora pedagógica de la Red Educativa SESI de la Federación de las Industrias de Rio de Janeiro

(2013 a 2016). Actúa en la profesión desde 2004 en el área de gestión educacional, coordinación y supervisión escolar, coordinación de proyectos educacionales para organizaciones sociales y empresas.

Investigadora del área de educación, procesos culturales singulares y políticas de diversidad en educación.

E-mail: suelynoronha@gmail.com

\section{Annette Kaltenbrunner Graf}

Licenciada en Ciencias Histórico Sociales por la Universidad Marcelino Champagnat (Lima - Perú), Magister en Gestión de Instituciones Educativas por la Universidad Antonio Ruíz de Montoya (Lima Perú). Cuenta con un diplomado de especialización en Gestión de la Calidad en Instituciones Educativas por la Pontificia Universidad Católica de Perú. Obtuvo un título de segunda especialidad en educación con mención en Formación Ciudadana y Cívica por la Universidad Peruana Unión. Participó durante 2013 como Docente Fortaleza en la implementación de las Rutas del Aprendizaje en la UGEL 05. Es docente en el área de Desarrollo Personal, Ciudadanía y Cívica de la Institución Educativa Fe y Alegría No.5 (San Juan de Lurigancho - Lima) desde 2003.

E-mail: annettekg@gmail.com

\section{Blanca Josefina Vallejos Saavedra}

Licenciada en Educación Secundaria en la especialidad de Historia y Geografía de la Universidad Federico Villarreal (Perú); Magíster en Gestión de Instituciones Educativas, por la Universidad Antonio 
Ruíz de Montoya (Perú). Estudios de maestría en Problemas de Aprendizaje en la Universidad Marcelino Champagnat (Perú). Es profesora de la Institución Educativa Fe y Alegría No.5 en San Juan de Lurigancho (Lima, Perú) en el área de Desarrollo Personal, Ciudadanía y Cívica. Participó del Programa de Alfabetización durante los años 2015 y 2016, como parte del Programa de Educación Básica Alternativa.

E-mail: vallejossaavedrablanca@gmail.com

\title{
RESUMEN
}

En los últimos veinte años, el Ministerio de Educación de Perú viene aplicando y acompañando la medición de logros de aprendizaje de los estudiantes de la educación básica por medio de evaluaciones nacionales e internacionales a gran escala. Estos estudios están llevando no sólo a conocer las causas de los logros de aprendizaje, sino al de tratar de revertir alguna situación no deseada. La investigación, en proceso, en la cual se basa este artículo, tiene como objetivo identificar de qué manera la inteligencia emocional influye en el rendimiento académico de estudiantes de nivel secundario de una institución educativa en Lima, Perú. Esta investigación fue orientada por la metodología cuantitativa y se utilizó de métodos estadísticos para inferir las relaciones entre la inteligencia emocional y el rendimiento académico de los alumnos en cada área curricular, a través de datos obtenidos por el test BarOn ICE: NA, y las calificaciones de los alumnos. Los resultados comprobaron la relación existente entre la inteligencia emocional y el desempeño escolar, mostrando que los componentes de la inteligencia emocional varían según el área curricular específica.

Palabras Claves: Inteligencia emocional, rendimiento académico, logro académico, investigación cuantitativa, análisis de grupos.

\begin{abstract}
In the last twenty years, the Peruvian Ministry of Education has been implementing and accompanying the measurement of learning achievements of students in basic education through large-scale national and international assessments. These studies are leading not only to know the causes of learning achievement, but to try to reverse some unwanted situation. The research, in process, on which this article is based, aims to identify how emotional intelligence influences the academic performance of secondary school students in an educational institution in Lima, Peru. This research was guided by the quantitative methodology and was used statistical methods to infer the relationships between emotional intelligence and academic performance of students in each curricular area, through data obtained by the BarOn ICE: NA test, and the qualifications of the students. The results verified the relationship between emotional intelligence and school performance, showing that the components of emotional intelligence vary according to the specific curricular area.
\end{abstract}

Keywords: Emotional intelligence, academic performance, academic achievement, quantitative investigation, clusters analysis.

\section{INTRODUCCIÓN}

En los últimos veinte años, el Ministerio de Educación de Perú (MINEDU), a través de la Unidad de Medición de la Calidad Educativa (creada en 1996), viene aplicando y acompañando con particular importancia la medición de los logros de aprendizaje de los estudiantes de la educación básica por medio 
de evaluaciones nacionales e internacionales a gran escala. Por otro lado, de acuerdo con Cueto (2007), prácticamente todos los países de Latino América habían creado hasta los años 1982 un sistema nacional de evaluación del rendimiento estudiantil.

Estos estudios de evaluación están llevando no sólo a conocer las causas de los logros de aprendizaje, sino al de tratar de revertir una situación no deseada. De acuerdo al Programa Estratégico de Logros del Aprendizaje (PELA), el MINEDU (2014) determina que el problema específico es el bajo logro de aprendizaje de los estudiantes de educación básica regular. En el mismo documento también muestra con respecto a los estudiantes de secundaria que las evaluaciones nacionales e internacionales hechas en los últimos años, revelan bajos niveles de aprendizaje. (MINEDU, 2014).

En el análisis de los bajos niveles de rendimiento escolar es común encontrar respuestas en investigaciones que indican en los alumnos la ausencia de un autoconcepto positivo, el poco control de impulsos, la falta de empatía; entre otros componentes que son considerados en este estudio como indicadores de la inteligencia emocional.

Numerosos estudios como los de Goleman (1995), Mayer y Salovey (1997), Castellano (2010), entre otros; han aseverado la importancia de la inteligencia emocional para el aprendizaje y el logro de un mejor rendimiento académico.

El aprendizaje es un proceso neural complejo, que lleva a la construcción de memorias. Lo que se aprende y después se olvida es como si nunca hubiera sucedido; el conjunto de cosas que recordamos constituye nuestra identidad. Las emociones y los afectos regulan el aprendizaje y la formación de memorias. Las personas se acuerdan mejor de lo que les despertó sentimientos positivos que de aquello que les despertó sentimientos negativos y recuerdan mal de lo que las dejó indiferentes. Las emociones contribuyen fuertemente a la motivación para que la persona aprenda; parecen dar color y sabor al que aprende. El aprendizaje puede ser pensado como un proceso de cambio, provocado por estímulos diversos, mediado por emociones, que puede venir o no a manifestarse en cambio en el comportamiento de la persona.

Existen diversos estudios sobre cómo la inteligencia emocional afecta a los estudiantes tanto dentro y fuera del contexto escolar. Autores como Brackett, Rivers, Shiffman, Lerner y Salovey (como se citó en Fernández y Ruiz, 2008), corroboran acerca de estos estudios, sobretodo en la influencia y efectos de la inteligencia emocional en niños y jóvenes estudiantes.

Los mismos autores citados arriba mencionan que las áreas fundamentales donde la inteligencia emocional perjudica o aporta a la vida escolar están relacionadas a las relaciones interpersonales, al bienestar psicológico, al rendimiento académico y a la aparición de conductas disruptivas. Se entiende que la capacidad de comprender nuestras emociones y la de los demás, poder corregir nuestros 
sentimientos, influye sobre el equilibrio psicológico de los estudiantes y esto redundará en su rendimiento escolar (Fernández y Ruiz, 2008).

Cada área curricular desarrolla competencias específicas en los alumnos. Al mismo tiempo, los alumnos desarrollan niveles de inteligencia emocional diferentes en cada área curricular. Por ejemplo, un alumno como alto nivel de inteligencia emocional puede obtener alto rendimiento académico en Matemáticas. Al mismo tiempo, este mismo alumno puede obtener un rendimiento académico medio en Comunicación, presentando para esta área un bajo nivel de inteligencia emocional. Es decir, los componentes de la Inteligencia Emocional (interpersonal, intrapersonal, adaptabilidad, manejo del estrés, estado de ánimo general) se relacionan con las competencias específicas de las áreas curriculares y con las diferentes inteligencias y habilidades individuales. Eso significa que la capacidad de resolución de problemas - que es una competencia fuertemente exigida en el área curricular de Matemáticas -, por ejemplo, donde el profesor, para el desarrollo de esta competencia, presenta al alumno como resolver diferentes tipos de problemas en los diferentes temas abordados en esta área; de esa forma, el área curricular de Matemáticas contribuye al desarrollo del componente de adaptabilidad de la Inteligencia Emocional para esta área, a diferencia del área de Artes o Comunicación donde presentan mayor contribución al desarrollo de otros componentes de la Inteligencia Emocional y menor al componente de adaptabilidad. Luego, el nivel de Inteligencia Emocional del alumno debe ser evaluado en relación a un área curricular específica.

Los componentes de la Inteligencia Emocional son comprendidos a partir de las siguientes definiciones: a) Interpersonal: capacidad para comprender las emociones de los demás, mostrándose hábil para ponerse en el lugar del otro. Incluye destrezas como la empatía, la responsabilidad social y el mantenimiento de relaciones interpersonales satisfactorias; b) Intrapersonal: capacidad para la comprensión y conocimiento de los estados emocionales de uno mismo. Incluye las habilidades para la autocomprensión emocional, para ser asertivo, para visualizarse a sí mismo de manera positiva, para autodirigirse y para sentirse seguro de sí mismo; c) Adaptabilidad: capacidad para enfrentar las emociones desagradables, autorregulándonos. Incluye las habilidades para ser flexibles, realistas y efectivos en el manejo de los cambios y ser eficaces para enfrentar los problemas cotidianos, evaluando la correspondencia entre lo subjetivo y lo objetivo; d) Manejo del estrés: capacidad para soportar eventos adversos, situaciones estresantes y fuertes emociones sin "desmoronarse", enfrentando activa y positivamente el estrés; controlar las emociones en situaciones críticas. Incluye la tolerancia al estrés y el control de los impulsos; e) Estado de ánimo general: capacidad para creer y estar seguro de sus propias habilidades y conocimientos. Significa que uno acepta sus experiencias emocionales únicas y excéntricas 
o culturalmente convencionales. Incluye la felicidad y el optimismo, tienen una apreciación positiva sobre las cosas o eventos.

A partir de esos aportes, se definió como objetivo de investigación (que se encuentra en proceso) identificar de qué manera influye la inteligencia emocional en el rendimiento académico de los estudiantes de tercer año de secundaria en una institución educativa en Lima (Perú) durante el año escolar 2017, visando medir el grado de inteligencia emocional de los estudiantes y correlacionar el resultado con los logros de aprendizaje.

Para obtener la medida de la inteligencia emocional fue utilizado el Inventario de BarOn ICE: NA (BarOn, 1997). En Perú, este modelo fue tomado en el estudio "La evaluación de la inteligencia emocional a través del inventario de BarOn (I-CE) en una muestra de Lima Metropolitana", elaborado por Nelly Ugarriza (2001). El inventario fue aplicado a varones y mujeres, a partir de los 15 años a más, en Lima Metropolitana. Esta investigación tuvo como uno de sus objetivos validar la adaptación del test BarOn ICE: NA para el contexto de la realidad de Lima.

Identificando la relación entre ambas variables (inteligencia emocional y rendimiento académico) esto permitiría a la escuela revertir la situación a través de programas que contribuyen a mejorar la inteligencia emocional y por ende poder elevar el rendimiento académico. De la misma forma podría servir de referente para poder intervenir a nivel de región y país en busca de mejores logros educativos.

El presente artículo se encuentra organizado en cinco sesiones: introducción, metodología, estudio de caso, análisis de datos y resultados, y conclusiones.

\section{METODOLOGÍA}

La investigación, en proceso, en la cual se basa este artículo fue orientada por la metodología cuantitativa y se utilizó de métodos estadísticos que medirán dos variables: inteligencia emocional, a través de un test, y rendimiento académico, a través de las calificaciones finales de los alumnos. Estas variables pueden ser definidas como:

- La inteligencia emocional como un conjunto de habilidades personales, emocionales, sociales y de destrezas que influyen en nuestra habilidad para adaptarnos y enfrentar a las demandas y presiones del medio (BarOn, 1997).

- El rendimiento académico como nivel de conocimientos demostrado en un área o materia, comparado con la norma de edad y nivel académico (Edel, 2003).

Fueron utilizados instrumentos estandarizados como es el caso del Inventario de BarOn ICE: NA (BarOn, 1997) según las adaptaciones realizadas por (Ugarriza, 2001; Ugarriza, N. y Pajares, L., 2003) para el contexto de la realidad de Lima. 
El test inventario de BarOn ICE: NA adaptado por Ugarriza está constituido por un cuestionario de sesenta preguntas que se relacionan a cinco componentes de la inteligencia emocional y a una variable de control (comúnmente denominada como una sexta componente de verificación), estas preguntas se encuentran distribuidas en el cuestionario de forma aleatoria. De acuerdo con BarOn (1997) y Ugarriza (2001), la inteligencia emocional está formada por cinco componentes: interpersonal, intrapersonal, adaptabilidad, manejo del estrés y estado de ánimo general; siendo que, como forma de validación de la veracidad de la medida obtenida para estos 5 componentes en los cuestionarios, fue creada una sexta variable (la impresión positiva), muchas veces también denominada componente, que busca evitar en lo posible la influencia de sesgos referidos a las respuestas de deseabilidad social. En la tabla 1 se presenta un ejemplo de las preguntas del cuestionario correspondiente a cada componente, indicando la cantidad de preguntas destinadas para cada componente.

Las respuestas del cuestionario se encuentran en escala Likert con cuatro opciones numéricas con significado presentado en la tabla 2:

Las calificaciones del rendimiento académico anual de los alumnos están organizadas por la nota final obtenida en cada área curricular correspondiente al año de 2017. En la tabla 3 se presentan las once áreas curriculares consideradas, con las respectivas competencias.

A partir de los cuestionarios y de las calificaciones finales de los alumnos, fue construida una base de datos en una estructura matricial o de tabla, de forma que cada columna representa una pregunta del cuestionario (60 columnas) y en las líneas se encuentran las respuestas a cada pregunta dadas por un alumno; de este modo se tienen tantas líneas como alumnos encuestados. A seguir, las preguntas fueron agrupadas en función al componente de la inteligencia emocional al que corresponden (como fue mostrado en el ejemplo de la tabla 1). Como cada línea corresponde a un mismo alumno, es posible asociar la calificación final de este alumno para cada área curricular, adicionando a la matriz más once columnas con estas calificaciones.

El siguiente paso fue realizar la limpieza de los datos, que consiste en retirar valores nulos, es decir, en retirar las líneas (un alumno encuestado) que presenten alguna pregunta sin respuesta o alguna calificación sin valor. Un procedimiento similar es aplicado para el caso de existir valores aberrantes en alguna línea de la matriz. De este modo se obtiene una matriz de datos válidos. 
Tabla 1: Ejemplo de pregunta por componente

\begin{tabular}{|c|c|c|}
\hline Componente & Pregunta & $\begin{array}{c}\text { Cantidad de } \\
\text { preguntas }\end{array}$ \\
\hline \multirow{3}{*}{$\begin{array}{l}\text { Interpersonal } \\
\text { (INTER) }\end{array}$} & $\begin{array}{l}\text { P002 - Soy muy bueno(a) para comprender cómo la gente se } \\
\text { siente }\end{array}$ & \multirow{3}{*}{12} \\
\hline & P005 - Me importa lo que les sucede a las personas & \\
\hline & P014 - Soy capaz de respetar a los demás & \\
\hline \multirow{3}{*}{$\begin{array}{l}\text { Intrapersonal } \\
\text { (INTRA) }\end{array}$} & P007 - Es fácil decirle a la gente cómo me siento & \multirow{3}{*}{6} \\
\hline & P017 - Puedo hablar fácilmente sobre mis sentimientos & \\
\hline & P028 - Es difícil hablar sobre mis sentimientos más íntimos & \\
\hline \multirow{3}{*}{$\begin{array}{l}\text { Adaptabilidad } \\
\text { (ADAP) }\end{array}$} & $\begin{array}{l}\text { P012 - Intento usar diferentes formas de responder las } \\
\text { preguntas difíciles }\end{array}$ & \multirow{3}{*}{10} \\
\hline & P016 - Es fácil para mí comprender las cosas nuevas & \\
\hline & P022 - Puedo comprender preguntas difíciles & \\
\hline \multirow{3}{*}{ Manejo del estrés (MAES) } & P003 - Puedo mantener la calma cuando estoy molesto & \multirow{3}{*}{12} \\
\hline & P006 - Me es difícil controlar mi cólera & \\
\hline & P011 - Sé cómo mantenerme tranquilo(a) & \\
\hline \multirow{3}{*}{$\begin{array}{l}\text { Estado de ánimo general } \\
\qquad(\mathrm{EAG})\end{array}$} & P009 - Me siento seguro(a) de mí mismo(a) & \multirow{3}{*}{14} \\
\hline & P013 - Pienso que las cosas que hago salen bien & \\
\hline & P019 - Espero lo mejor & \\
\hline \multirow{3}{*}{$\begin{array}{l}\text { Impresión positiva } \\
\text { (IMPO) }\end{array}$} & P008 - Me gustan todas las personas que conozco & \multirow{3}{*}{6} \\
\hline & P018 - Pienso bien de todas las personas & \\
\hline & P027 - Nada me molesta & \\
\hline
\end{tabular}

Tabla 2: Escala de respuestas del cuestionario

\begin{tabular}{|c|c|}
\hline Escala & Descripción \\
\hline 1 & Muy rara vez \\
\hline 2 & Rara vez \\
\hline 3 & A menudo \\
\hline 4 & Muy a menudo \\
\hline
\end{tabular}


Tabla 3: Áreas curriculares y competencias

\begin{tabular}{|c|c|c|}
\hline Área curricular & Que se enseña & Competencias \\
\hline Matemática & $\begin{array}{l}\text { Matemáticas, Estadística, } \\
\text { Relaciones lógicas, Algebra, } \\
\text { Aritmética }\end{array}$ & $\begin{array}{l}\text { Actúa y piensa matemáticamente en situaciones de cantidad; actúa y } \\
\text { piensa matemáticamente en situaciones de regularidad, equivalencia } \\
\text { y cambio; actúa y piensa matemáticamente en situaciones de forma, } \\
\text { movimiento y localización; actúa y piensa matemáticamente en } \\
\text { situaciones de gestión de datos e incertidumbre. }\end{array}$ \\
\hline $\begin{array}{l}\text { Persona, Familia } \\
\text { y Relaciones } \\
\text { Humanas }\end{array}$ & \begin{tabular}{|l|} 
Psicología \\
Ética \\
Adolescencia \\
Autoconocimiento \\
Sexualidad y genero \\
Vínculo familiar \\
\end{tabular} & Afirma su identidad y se desenvuelve éticamente. \\
\hline Comunicación & $\begin{array}{l}\text { Comprensión lectora } \\
\text { Redacción de textos } \\
\text { Gramática } \\
\text { Ortografía } \\
\text { Lenguaje audiovisual } \\
\text { Literatura }\end{array}$ & $\begin{array}{l}\text { Comprensión de textos orales, expresión oral, comprensión y } \\
\text { producción de textos escritos e interacción con expresiones } \\
\text { literarias. }\end{array}$ \\
\hline $\begin{array}{l}\text { Educación } \\
\text { Religiosa }\end{array}$ & $\begin{array}{l}\text { Conciencia moral cristiana } \\
\text { Testimonios de vida } \\
\text { La biblia } \\
\text { Principales fiestas religiosas } \\
\text { cristianas } \\
\text { La presencia de Dios en la } \\
\text { naturaleza } \\
\end{array}$ & Comprensión doctrinal cristiana y discernimiento de fe. \\
\hline Educación Física & $\begin{array}{l}\text { Condición física y salud } \\
\text { Motricidad, ritmo y expresión } \\
\text { Juegos y deportes } \\
\end{array}$ & $\begin{array}{l}\text { Comprensión y desarrollo de la corporeidad y la salud, dominio } \\
\text { corporal y expresión creativa, convivencia e interacción sociomotriz. }\end{array}$ \\
\hline $\begin{array}{l}\text { Historia, } \\
\text { Geografía y } \\
\text { Economía }\end{array}$ & $\begin{array}{l}\text { Historia del Perú } \\
\text { Historia Universal } \\
\text { Geografía del Perú y del } \\
\text { Mundo } \\
\text { Economía }\end{array}$ & $\begin{array}{l}\text { Construye interpretaciones históricas, actúa responsablemente en el } \\
\text { ambiente, actúa responsablemente respecto a los recursos } \\
\text { económicos y participa en asuntos públicos para promover el bien } \\
\text { común }\end{array}$ \\
\hline $\begin{array}{l}\text { Formación } \\
\text { Ciudadana y } \\
\text { Cívica }\end{array}$ & $\begin{array}{l}\text { Participación en proyectos } \\
\text { ciudadanos que buscan el bien } \\
\text { común } \\
\text { Derechos Humanos } \\
\text { Estado y democracia } \\
\text { Valores cívicos y } \\
\text { democráticos } \\
\text { Cultura de paz } \\
\end{array}$ & $\begin{array}{l}\text { Convive respetándose a sí mismo y a los demás y participa en } \\
\text { asuntos públicos para promover el bien común. }\end{array}$ \\
\hline Arte & $\begin{array}{l}\text { Artes plásticas } \\
\text { Historia del arte } \\
\text { Música, Danza, Teatro } \\
\end{array}$ & Expresión artística y apreciación artística. \\
\hline Inglés & $\begin{array}{l}\text { Redacción en ingles } \\
\text { Pronunciación } \\
\text { Lectura en ingles } \\
\end{array}$ & Expresión y comprensión oral, Comprensión y producción de textos. \\
\hline $\begin{array}{l}\text { Educación para el } \\
\text { Trabajo }\end{array}$ & $\begin{array}{l}\text { Talleres de: } \\
\text { Ofimática, Electrónica } \\
\text { Industria alimentaria } \\
\text { Industria del vestido } \\
\text { Carpintería. } \\
\text { Gestión empresarial } \\
\end{array}$ & $\begin{array}{l}\text { Gestión de procesos, ejecución de procesos; comprensión y } \\
\text { aplicación de tecnologías }\end{array}$ \\
\hline $\begin{array}{l}\text { Ciencia, } \\
\text { Tecnología y } \\
\text { Ambiente }\end{array}$ & $\begin{array}{l}\text { Mundo físico, tecnología y } \\
\text { ambiente } \\
\text { Salud integral, tecnología y } \\
\text { sociedad } \\
\text { Química orgánica e inorgánica } \\
\text { Biología, Física }\end{array}$ & $\begin{array}{l}\text { Indaga mediante métodos científicos, situaciones que puedan ser } \\
\text { investigadas por la ciencia; explica el mundo físico basado en } \\
\text { conocimientos científicos; diseña y produce prototipos tecnológicos, } \\
\text { para resolver problemas de su entorno; construye una posición } \\
\text { crítica sobre la ciencia y la tecnología en sociedad. }\end{array}$ \\
\hline
\end{tabular}


A continuación, como los datos presentan escalas diferentes (las respuestas al cuestionario varían de 1 a 4, mientras que las calificaciones varían de 0 a 20), se procede a padronizar la escala de datos para cada columna, de forma que todas los datos tomen valores de 0 a 20, la ecuación 1 presenta la función de padronización utilizada.

$$
Y_{i}=a+\frac{b *\left(x_{i}-x_{\min }\right)}{\left(x_{\max }-x_{\min }\right)}
$$

Donde $Y_{i}$ es un dato padronizado, $a$ es el menor valor de la nueva escala (en este caso 0 ), $b$ es el mayor valor de la nueva escala (en este caso 20 ), $x_{i}$ es el dato que desea padronizar, $x_{\min }$ es el menor valor de la escala de la columna ( i la columna corresponde a pregunta es 1 , se corresponde a calificación es 0 ) y $x_{\max }$ es el mayor valor de la escala de la columna (si la columna corresponde a pregunta es 4 , se corresponde a calificación es 20).

Son obtenidas las estadísticas descriptivas de cada columna y sus distribuciones a través de diagramas box-plot.

A seguir se procedió a la simplificación de la matriz a través de la obtención de las componentes de la inteligencia emocional (interpersonal, intrapersonal, adaptabilidad, manejo del estrés, estado de ánimo general y la variable de verificación: impresión positiva). Estas seis nuevas columnas se forman por la agregación de los valores de las preguntas que conforman cada componente. Estas seis columnas también son padronizadas en la escala de 0 a 20 considerando la proporción con respecto al número de preguntas que forman cada componente.

De esta forma, se construye una nueva matriz de datos simplificada, formada por las once columnas de las calificaciones de las áreas curriculares, más las seis columnas de los componentes de la inteligencia emocional (total 17 columnas).

A partir de esta matriz de datos simplificada se procede a realizar el análisis de relaciones entre las calificaciones de cada área curricular con los componentes de la inteligencia emocional, para lo cual se realiza un análisis de agrupamiento (clustering). Para ayudar en la interpretación de los resultados del análisis de grupos, fue definida una escala Likert para los componentes, tabla 4:

Tabla 4: Escala Likert para los componentes de IE.

\begin{tabular}{|c|c|}
\hline Escala & Descripción \\
\hline 0 a 5 & Muy bajo \\
\hline 5 a 8 & Bajo \\
\hline 9 a 12 & Medio bajo \\
\hline 13 a 15 & Medio \\
\hline 16 a 18 & Alto \\
\hline 18 a 20 & Sobre saliente \\
\hline
\end{tabular}




\section{ESTUDIO DE CASO}

La Institución Educativa investigada es Fe y Alegría No. 5, ubicada en el distrito de San Juan de Lurigancho, departamento de Lima (Perú). Los barrios de este distrito se caracterizan por la gran cantidad de comercios formales e informales, tal como por presentar alto índice de inseguridad ciudadana. Por lo general, los padres de familia se dedican al comercio de microempresa o por estar empleados en instituciones, negocios o fábricas. Los estudiantes pertenecen en su gran mayoría a familias disfuncionales (padres separados, madres solteras o viven con algún familiar).

La Institución investigada cuenta con 1.100 estudiantes de educación secundaria que comprende los cinco grados con seis secciones de primero y segundo año, y siete secciones de tercero, cuarto y quinto año. La cual se caracteriza por una preocupación en la búsqueda de brindar una calidad educativa de acuerdo a la realidad y tomando en cuenta las necesidades e intereses de los estudiantes. Para tal fin, cada año se realiza un diagnóstico con el objetivo de responder a la problemática del entorno y de la escuela. Durante los últimos cuatro años consecutivos, el diagnóstico apuntó entre los principales problemas las agresiones físicas y verbales entre pares, así como el bajo rendimiento académico.

La Institución fue elegida para la investigación debido a la aplicación continua de los diagnósticos especializados, así como a las problemáticas presentes en el ambiente escolar relacionada al tema de la Inteligencia Emocional, así como a la facilidad en obtener datos de la Institución y aplicar encuestas en la misma.

Para medir la inteligencia emocional de los alumnos se aplicó el cuestionario del inventario de BarOn ICE-NA a un total de 169 adolescentes entre 14 y 17 años, correspondientes a las siete secciones de tercer año, en sus respectivas aulas; aplicado entre el 15 y 21 de diciembre del 2017.

La elección del tercer año responde al objetivo de la investigación que es seguir acompañando a los alumnos en el siguiente año e intervenir con alternativas viables que visen la mejora del nivel de inteligencia emocional y del incremento del rendimiento académico de los alumnos.

Los estudiantes recibieron el test con la breve explicación de cómo deberían marcar los ítems de acuerdo a los rangos establecidos. Se les indicó que no había un tiempo límite, sin embargo, todos terminaron sin exceder los 45 minutos.

Las calificaciones del rendimiento académico anual de los alumnos se obtuvieron mediante los registros del SIAGIE (Sistema de Información de Apoyo a la Gestión de la Institución Educativa), del Ministerio de Educación de Perú. 


\section{ANÁLISIS DE DATOS}

Siguiendo la metodología, se realizó la limpieza de los datos obtenidos en las encuestas; los investigadores optaron por considerar datos no válidos aquellos correspondientes a encuestas con preguntas no respondidas. Así que, de los 169 alumnos que respondieron a la encuesta, 127 (71 hombres y 56 mujeres) fueron considerados en el presente análisis. Las 42 encuestas con preguntas no respondidas fueron excluidas.

Se construyó la matriz de datos con los resultados de las encuestas y las calificaciones de los alumnos, luego se realizó la padronización de datos y la creación de las columnas de componentes y la simplificación de la matriz de datos. A partir de esta matriz de datos fue realizado el análisis de clusters (grupos) para cada área curricular y los componentes de Inteligencia Emocional (IE), buscando responder la pregunta ¿Es la IE un factor influyente en el rendimiento académico de los estudiantes de tercer año de secundaria en la Institución Educativa Fe y Alegría No. 5 durante el año escolar 2017?

\section{DISCUSIÓN DE RESULTADOS}

A seguir se describen las relaciones encontradas a partir de los resultados del análisis de clusters para las áreas curriculares de Matemática y Educación Física, interpretados con apoyo de la escala Likert para los componentes de IE presentada en la tabla 4.

Así, para el área curricular de matemáticas se observó en promedio los siguientes perfiles o patrones de los componentes de la IE sobre el rendimiento académico de los alumnos, tabla 5.

Tabla 5: Perfil promedio de los componentes de IE en el área curricular de Matemática

\begin{tabular}{|c|c|}
\hline Componentes & Escala \\
\hline Interpersonal & Medio \\
\hline Intrapersonal & Bajo \\
\hline Adaptabilidad & Medio bajo \\
\hline Manejo de estrés & Bajo \\
\hline Estado de ánimo general & Medio \\
\hline
\end{tabular}

Como se observa en al tabla 5, el componente interpersonal, independientemente de las notas de los alumnos, presentó un nivel medio alcanzado para esta área; esto podría darse debido a la creencia de los alumnos de matemática no ser fácil. Ellos tienden a apoyarse y, muchas veces, a trabajar en equipo, lo que justamente ayuda mucho a los estudiantes en levantar sus promedios; en consecuencia, los estudiantes han ido desarrollando las habilidades que necesitan para interactuar. Pero, esa interacción no se aprecia de forma permanente.

El componente intrapersonal muestra un nivel bajo que podría ser casi natural por el hecho de estar atravesando una etapa muy crítica como adolescente, en el cual le cuesta, todavía, comprenderse a sí mismo y controlar sus emociones. 
Para el componente adaptabilidad, que comprende básicamente las habilidades para adaptarse a las diferentes situaciones se evidencia un nivel medio bajo prácticamente de forma general, sólo destacando que los estudiantes con más altas calificaciones poseen un nivel mayor de adaptabilidad (nivel medio), lo que les permitiría ser más creativos para encontrar soluciones a los problemas, especialmente en el área. Mientras que, los alumnos con menos nota podrían tener mayor adaptabilidad por el hecho de siempre obtener bajas calificaciones en el área los empuja a la tendencia de resignarse y adaptarse a esa realidad que, para ellos, siempre fue así.

Sobre el componente de manejo del estrés los estudiantes presentan un nivel bajo que se evidencia por el hecho de que, en su mayoría, los estudiantes suelen dejar para último momento el cumplimiento de tareas, lo que podría generar mayor estrés, que ellos no alcanzan a manejarlo y equilibrarlo.

El componente de estado de ánimo general presentó un nivel medio de forma general, esto podría explicarse debido a que se observa en el grupo de estudiantes que alcanzan un promedio mayor a 14 la satisfacción de ser bueno en el área, y esto le genera complacencia al ser considerado por una gran mayoría como una de las áreas más importantes en la escuela; mientras en el grupo que alcanza un promedio menor, por la actitud de conformismo que presentan, se encuentran satisfechos con el solo hecho de aprobar en el área.

A continuación, se describe el perfil de los componentes de IE para el área curricular de Educación Física observado del análisis de clusters.

El componente interpersonal en el área Educación Física presentó un nivel medio para todas las calificaciones de los alumnos. Como en el deporte requieren de una interacción entre pares y se podría deducir que son conscientes que para el juego en equipo requieren de empatía y capacidad de no herir al otro.

El componente intrapersonal para esta área muestra un nivel bajo para la mayoría de los alumnos, excepto en los estudiantes con calificación más baja (con nivel medio bajo). Esto podría ser casi natural a la etapa de adolescencia en la que se encuentran los alumnos; en la cual les cuesta comprenderse a sí mismos y controlar sus emociones. Ya para los estudiantes con calificación más baja, estos reconocen sus limitaciones que impiden un mayor desempeño en los ejercicios físicos tomando sus bajas notas con tranquilidad.

Las habilidades que componen la adaptabilidad y que se relacionan con el área de Educación Física, no son bien desarrolladas en los estudiantes. Se mantienen en un nivel medio bajo y, únicamente los estudiantes con la nota más baja llegan a un nivel medio. Ellos se han adecuado al nivel de exigencia en el área y son realistas de sus capacidades que impiden realizar los ejercicios de manera satisfactoria. 
El componente manejo del estrés está en un nivel bajo. La educación física debe contribuir a bajar el nivel de estrés, pero, los estudiantes no saben aprovechar este espacio para equilibrarlo.

Se observa que el componente estado de ánimo general alcanza el nivel medio entre todos los estudiantes sin importar el rango de sus calificaciones. Es inherente al área de Educación Física que los estudiantes cambien el uniforme a ropa deportiva, más cómoda y, también a un ambiente, el patio con las canchas, que probablemente genera una expectativa y levanta el ánimo en los adolescentes.

Todavía son pocos los trabajos que abordan el problema de relacionar la IE con el rendimiento académico, siendo que varios utilizan la escala TMMS (Trait-Meta Mood Scale) (Salovey y Mayer, 1995) que evalúa el metaconocimiento de los estados emocionales mediante un cuestionario de 48 preguntas, que busca medir las destrezas con las que podemos ser conscientes en nuestras propias emociones, así como, de nuestra capacidad para regularlas. El TMMS contiene tres dimensiones claves de la IE (Percepción emocional, Comprensión de sentimientos y Regulación emocional). Uno de los relevantes es el trabajo de Vissupe et al. (2017) que analiza la relación de la Inteligencia Emocional (IE) con el rendimiento académico de preadolescentes de 12 a 14 años con bajo rendimiento académico en una escuela en Angola; para lo cual se aplicó el Trait Meta-Mood Scale (TMMS), Salovey (1995), buscando relacionar la IE con el rendimiento académico de los alumnos. No incluyen a los alumnos con alto rendimiento. De los resultados de las encuestas del TMMS fue realizado un análisis de estadística descriptiva, donde muestra la frecuencia de la incidencia de bajo desempeño para cada categoría (dificultades en el aprendizage, repitencia, abandono escolar y número de asignaturas reprobadas). De los resultados, se concluyó que la IE, para el grupo de estudio, presentaba una adecuada percepción y compresión emocional, y con mayores dificultades para la regulación emocional. El trabajo de Vissupe et al. (2017) a pesar de tener relación con la presente investigación, se diferencia porque esta investigación se analizó en niños con edades diferentes (adolescentes entre 14 y 17 años) usando una adaptación del test de Bar-On por permitir extraer cuatros componente de IE más una variable de validación, mientras que el test TMMS tiene apenas tres dimensiones.

En el estudio cuantitativo transversal correlacional realizado en Portugal (Silva, 2012) tuvo como objetivo correlacionar la inteligencia emocional con variables del rendimiento escolar de 129 estudiantes del 1ro Ciclo de Educación Básica, entre 6 y 13 años. Se utilizó los instrumentos para la retención de datos: Información Sociodemográfica e Educativa, Matrices Coloridas de Raven, Cuestionario de Inteligencia Emocional de Bar-On y o Test of Emotional Comprehension. Los resultados destacan que solo algunos aspectos de la Inteligencia emocional se relacionan con algunas variables del rendimiento escolar. El trabajo de (Silva, 2012) apuntó constataciones interesantes al usar el test de Bar-On y o test of Emotional Comprehension, motivando nuestro investigación en el uso de la adaptación del test de Bar- 
On y análisis de clusters para identificar la influencia de las componentes de la IE en el rendimiento académico en cada área curricular.

\section{CONCLUSIONES}

Este artículo presenta la investigación realizada en alumnos de tercer año de educación secundaria en la Institución Educativa Fe y Alegría No. 5 para determinar y medir la influencia de la Inteligencia Emocional (IE) en el rendimiento académico por área curricular.

Este trabajo permitió definir una escala para medir la relación entre el nivel de los componentes de la IE y el rendimiento académico de los estudiantes.

Se comprobó que los cuatro niveles utilizados para medir el desempeño del alumno, en cuanto al cumplimiento de las competencias en las áreas curriculares (no logró, en proceso, logró y logro destacado), no son suficientes cuando se desea medir la influencia de cada componente de la IE en el rendimiento por área curricular, por lo que fue creada una escala Likert de seis niveles (muy bajo, bajo, medio bajo, medio, alto y sobresaliente).

Cuando analizada la influencia de cada componente de la IE en el área de Educación Física, esta se mostró similar en todos los alumnos, indicando que la Inteligencia Emocional en esta área curricular tiene el mismo grado de influencia en el rendimiento académico sobre todos los alumnos.

Los componentes de la IE en el área de Matemática influencian de forma diferente a los alumnos, siendo los componentes interpersonal y estado de ánimo general los que más se destacan, en especial para los alumnos con las más altas y más bajas notas (en ambos casos con nivel medio), mostrando tranquilidad en cuanto a sus calificaciones y la necesidad o presión del ambiente para apoyarse. Algo similar ocurre con el componente de adaptabilidad y el mismo grupo de alumnos (con más altas y más bajas calificaciones).

En general se observó que el componente de manejo del estrés de los alumnos de tercer año es bajo, independientemente del área curricular analizada.

Asimismo se comprobó que el componente intrapersonal es bajo en todos los alumnos de tercer año. Aquí se levanta la hipótesis que esto puede ser en gran parte debido a la etapa de adolescencia en la que se encuentran los alumnos. Esta constatación puede ser de utilidad para la escuela, la cual al definir actividades escolares que los ayuden a mejorar los niveles de esa componente podrá generar más confianza y seguridad en los alumnos, generando, así, una posible mejora sobre el rendimiento.

Adicionalmente, la metodología de análisis de datos aquí propuesta permite identificar el nivel de cada componente de la IE de forma individual para cada alumno. El análisis de este tipo de información 
permite a la escuela o profesor tomar medidas específicas por alumno que contribuyan a la mejora del rendimiento. 


\section{REFERENCIAS}

BarOn, R. (1997). Development of the Baron EQ-I: A measure of emotional and social intelligence. 105th Annual Convention of the American Psychological Association in Chicago.

Castellano, J. (2010). Inteligencia emocional y comprensión lectora en alumnos de sexto grado de primaria de la red $\mathrm{N}^{\circ} 4$ distrito del Callao (Tesis de Maestría). Universidad San Ignacio de Loyola, Lima, Perú.

Cuadras, C. (2010). Nuevos métodos de análisis multivariante. Barcelona, Spain: CMC Editions.

Cueto, S. (2007). Las evaluaciones nacionales e internacionales de rendimiento escolar en el Perú: balance y perspectivas. GRADE: Investigación, políticas y desarrollo en el Perú. p. 405-455. Recuperado de http://www.grade.org.pe/upload/publicaciones/archivo/download/pubs/InvPolitDesarr-10.pdf

Edel, R. (2003). El Rendimiento académico: Concepto, investigación y desarrollo. REICE - Revista Electrónica Iberoamericana sobre Calidad, Eficacia y Cambio en Educación, 1 (2). Recuperado de https://revistas.uam.es/index.php/reice/article/view/5354/5793

Fernández-Berrocal, P. y Extremera, N. (2005). La Inteligencia Emocional y la educación de las emociones desde el Modelo de Mayer y Salovey. Revista Interuniversitaria de Formación del $\begin{array}{lllll}\text { Profesorado, } & 19 & \text { (3), } & \text { 63-93. } & \text { Recuperado }\end{array}$ http://emotional.intelligence.uma.es/documentos/pdf61modelo_de_mayer_salovey.pdf Goleman, D. (1995). Inteligencia emocional. Barcelona, España: Editorial Kairos, S.A.

Jambu, M., Lebeaux, M. (1985). Cluster analysis and data analysis. Journal of the American Statistical Association, 80 (390), 482.

Martínez-Otero Pérez, V. (2009). Diversos condicionantes del fracaso escolar en la educación secundaria. Revista Iberoamericana de Educación, 51, 67-85. Recuperado de http://rieoei.org/rie51a03.pdf

Mayer \& Salovey (1997). What is Emotional Intelligence? Chapter 1 (3-31) in Emotional development and emotional intelligence: Educational implications. New York: Basicbook - A dimension of harper Collins Publishers.

MINEDU (2009). Diseño Curricular Nacional de Básica Regular. Lima: World Color Perú, S.A. MINEDU (2014). Ministerio de Educación, Programa presupuestal "Logros de aprendizaje de los estudiantes de educación básica regular-pela” 2014-2016. Recuperado de http://www.minedu.gob.pe/opyc/files/Anexo02pela2014junio.pdf

MINEDU (2016). Educación Básica Regular, Programa curricular de la Educación Secundaria. Recuperado de http://www.minedu.gob.pe/curriculo/pdf/programa-curricular-educacion-secundaria.pdf

Salovey, P., Mayer, J., Goldman, S., Turvey, C., \& Palfai, T. (1995). Emotional attention, clarity, and repair: exploring emotional intelligence using the Trait Meta-Mood Scale. In J.W. Pennebaker (Ed.).

Emotion, disclosure, and health (pp. 125-154). Washington, DC: American Psychological Association. Recuperado de http://dx.doi.org/10.1037/10182-006

Silva, E. (2012). A Relação entre inteligencia Emocional e o Rendimiento Escolar em Crianças do $1^{\circ}$ Ciclo do Ensino Básico da R. A. M (tesis de maestría). Universidade da Madeira. Portugal. 
Ugarriza, N. (2001). La evaluación de la inteligencia emocional a través del inventario de BarOn (I-CE) en una muestra de Lima Metropolitana. Red de Revistas Científicas de América Latina, el Caribe, España y Portugal, 4. 129-160. Recuperado de http://www.redalyc.org/articulo.oa?id=147118178005

Ugarriza, N. y Pajares, L. (2003). Adaptación y estandarización del inventario de inteligencia emocional de BarOn ICE - NA en niños y adolescentes. Manual técnico Lima, Perú: TEA Ediciones.

Vissupe, M., Jacob, A., Gallo, L. y Morales, V. (2017). Inteligencia emocional: Su relación con el rendimiento académico en preadolescentes de la Escuela 4 de Abril, de Io ciclo, Angola. Revista

Educación 41 (2). Recuperado de https://revistas.ucr.ac.cr/index.php/educacion/article/view/22713/pdf 